\title{
Al and Si oxyanions impact on the structural organization of $\mathrm{Fe}-\mathrm{OM}$ nanoaggregates
}

DELPHINE VANTELON ${ }^{1}$, ANTHONY BEAUVOIS ${ }^{2}$, MELANIE DAVRANCHE ${ }^{3}$ AND JACQUES JESTIN ${ }^{4}$

${ }^{1}$ Synchrotron SOLEIL

${ }^{2}$ PHENIX, Sorbonne Université

${ }^{3}$ Univ Rennes, CNRS, Géosciences Rennes, UMR 6118

${ }^{4}$ Institut Laue-Langevin

Presenting Author: delphine.vantelon@synchrotron-soleil.fr

Natural colloids composed of iron $(\mathrm{Fe})$ and organic matter (OM) are a key factor controlling the mobility of metallic pollutants due to their high adsorption capacity, a consequence of their high density of binding sites. The physico-chemical conditions under which $\mathrm{Fe}-\mathrm{OM}$ nano-aggregates are formed influence their structural organization, and more particularly the speciation of $\mathrm{Fe}$. In this study, we probe the influence of two major elements of natural systems: aluminum (Al) and silicon (Si). Al is known to have a high affinity with $\mathrm{OM}$ but also easily enters the structure of $\mathrm{Fe}$ hydroxides. $\mathrm{Si}$, on the other hand, is known to inhibit the growth and crystallinity of Fe hydroxides, although the mechanisms remain unknown. $\mathrm{Al}$ and $\mathrm{Si}$ are therefore expected to influence the Fe-OM nano-aggregates organization and to have an impact on the Fe speciation.

$\mathrm{Fe}-\mathrm{OM}-\mathrm{Al} / \mathrm{Si}$ nano-aggregates, mimicking environmental ones, have been synthesized with different $\mathrm{Fe} / \mathrm{OM}$ and oxyanion/ $\mathrm{Fe}$ ratios. In these systems, the $\mathrm{Fe}$ speciation is complex and variable, depending on the $\mathrm{Fe}$ and oxyanion content relative to $\mathrm{OM}$. The $\mathrm{Fe}$ phases appear to be composed of oligomers and ferrihydrite-like nanoparticles (Fh-Np), both integrated in the OM matrix. The Fh-Np form a fractal network whose organization is controlled by the OM. As Fe/OM increases, the oligomer content decreases in favor of the Fh- Np, which increases in size. By adding $\mathrm{Al}$ or $\mathrm{Si}$, this phenomenon may strongly differ. Al, forming oligomers and bound to both $\mathrm{Fe}$ and OM, clearly allows the growth of the Fh-Np/oligomer ratio, the Fh-Np size and the whole nano-aggregates structure. On the contrary, $\mathrm{Si}$, bound to $\mathrm{Fe}$, has the exact opposite effect. These differences result from the different interactions between $\mathrm{Al}$ and $\mathrm{Si}$ and the components of the Fe-OM nano-aggregates.

These results clearly highlight the antagonist effect of the major elements, $\mathrm{Al}$ and $\mathrm{Si}$, on the structural organization of $\mathrm{Fe}$ OM colloids. They impact all the levels of organization: the Fe speciation and the OM and Fh-Np arrangement. This structural variability has a direct consequence on the ability of Fe-OM nano-aggregates to trap and transport pollutants in the hydrographic network. 